\title{
Impact of thymidylate synthase protein expression on efficacy of chemotherapy in advanced lung cancer patients
}

\author{
YOSHIKO NAKAGAWA, TETSUO SHIMIZU, NORIAKI TAKAHASHI and SHU HASHIMOTO \\ Division of Respiratory Medicine, Department of Internal Medicine, \\ Nihon University School of Medicine, Tokyo 173-8610, Japan
}

Received August 23, 2012; Accepted February 4, 2013

DOI: $10.3892 / \operatorname{mco} .2013 .94$

\begin{abstract}
Advanced lung cancer is primarily treated with platinum combination chemotherapy, however, its prognosis remains poor. The aim of this study was to examine the correlation between the expression of thymidylate synthase (TS) in cancer tissues and the efficacy of chemotherapy in patients with advanced lung cancer in order to clarify the role of TS in the overall response. In total, 120 patients diagnosed with lung cancer between June 2004 and December 2010 at Nihon University Hospital, Tokyo, Japan, were included in this study. Cancer tissue specimens were obtained from the included patients by surgery or bronchofiberscopy prior to treatment. The expression of TS protein was evaluated using specimens immunostained with anti-TS antibody and $\mathrm{H}$-scoring. TS protein expression tended to be higher in smokers compared with non-smokers. Overall survival (OS) (median value) was significantly prolonged in the low TS expression group compared with the high TS expression group. More favorable therapeutic effects were observed in the high TS expression group compared with the low TS expression group, when carboplatin + paclitaxel combined chemotherapy (CbPac therapy) was used. When the therapeutic effects were compared between CbPac therapy and carboplatin + pemetrexed combined chemotherapy (CbPem therapy) in the high TS expression group, prolongation of OS (median value) was observed with CbPac therapy. The present study suggests that TS protein expression is a critical factor in determining the efficacy of $\mathrm{CbPac}$ therapy in lung cancer. CbPac therapy is more effective when TS protein is highly expressed in lung cancer tissue.
\end{abstract}

\section{Introduction}

Lung cancer has been the leading cause of mortality from cancer in Japan since 1998, with 50,000 deaths annually.

Correspondence to: Dr Tetsuo Shimizu, Division of Respiratory Medicine, Department of Internal Medicine, Nihon University School of Medicine, 30-1 Oyaguchi-Kamichou, Itabashi-ku, Tokyo 173-8610, Japan

E-mail: shimizu.tetsuo@nihon-u.ac.jp

Key words: thymidylate synthase, paclitaxel, pemetrexed, immunohistochemistry
Anti-smoking measures as a primary prevention strategy, early diagnosis through health-check campaigns, as well as advances in surgical therapy, chemotherapy and radiation therapy have improved overall prognosis. However, the 5-year survival rate remains at only $\sim 13 \%$. Compared with other types of cancer, sufficient lung cancer therapeutic results have yet to be achieved. Lung cancer is frequently diagnosed in advanced stages, thus, the primary treatment mainly comprises platinum combination chemotherapy. Carboplatin + paclitaxel combination chemotherapy (CbPac therapy) is a typical regimen for non-small cell lung cancer (NSCLC), and is widely used in clinical practice.

The occurrence, progression and metastasis of cancer involve various gene and protein abnormalities. In lung cancer, mutations of the epidermal growth factor receptor (EGFR) and KRAS genes as well as abnormalities in protein expression have been previously reported (1-3). Recently, the correlation of these gene mutations and protein expression abnormalities with the therapeutic effects has been extensively studied, and EGFR gene mutation was identified as a predictive factor of the therapeutic effect of EGFR tyrosine kinase inhibitors $(4,5)$.

Thymidylate synthase (TS) is a key enzyme in DNA synthesis and cell growth that has been suggested to be involved in malignancy (6-8). Moreover, it is a main target protein of antimetabolites, such as the anticancer agents pemetrexed (Pem) (9) and S-1 (10), demonstrating clinical efficacy in NSCLC. In colon (11), breast (12) and pancreatic cancer (13), an association between TS expression in cancer tissue, antitumor effects of chemotherapy and prognosis has been suggested. In lung cancer, a correlation between TS expression and prognosis has been suggested in early cancer (14). A limited number of studies have examined TS expression in advanced lung cancer, however, its impact on clinical effects remains to be determined. In particular, whether TS expression in cancer tissue is involved in the efficacy of CbPac therapy and prognosis remains to be elucidated. The aim of this study was to examine TS expression in cancer tissues obtained from patients with advanced lung cancer, and investigate the correlation between the expression rate and therapeutic effects and prognosis.

\section{Patients and methods}

Patients. In total, 120 patients diagnosed with lung cancer at Nihon University Hospital (Tokyo, Japan) between June 2004 
Table I. Patient characteristics and treatment methods.

\begin{tabular}{l} 
Characteristics \\
\hline Age (years) \\
Mean (range) \\
Gender \\
Male \\
Female
\end{tabular}

Value, n $(\%)$

Histology

Adenocarcinoma

Squamous cell carcinoma

Non-small cell carcinoma

Large cell carcinoma

Small cell carcinoma

EGFR mutation

Positive

Negative

Unknown

ECOG performance status

0

1

2

3,4

Stage of disease

I, II

IIIA

IIIB

IV

Smoking status

Former/current smoker

Non-smoker

Unknown

TS protein expession

High

Low

$66(55.0)$

$54(45.0)$

Treatment

Operation

Thoracic radiotherapy

Chemotherapy plus radiotherapy

Chemotherapy

$\mathrm{CbPac}$

$50(41.7)$

Gemcitabine

Cisplatin + S-1

Docetaxel

Gefitinib

Carboplatin + Irinotecan

Carboplatin + gemcitabine

Carboplatin + PMT

Cisplatin + VNR

Irinotecan

PMT
Table I. Continued.

Characteristics

Value, n (\%)

Chemotherapy

TS1

4 (3.3)

VNR

Others

Total no. of patients $=120$. EGFR, epidermal growth factor receptor; ECOG, Eastern Cooperative Oncology Group; TS, thymidylate synthase; CbPac, carboplatin + paclitaxel combined chemotherapy; PMT, Pemetrexed; VNR, vinorelbine.

and December 2010 were included in this study. Cancer tissue specimens were obtained from the included patients prior to treatment. The method of this study was approved by the ethics committee of Nihon University School of Medicine. Written informed consent was obtained from each subject. Cancer tissue specimens were collected by surgical procedure or bronchofiberscopic biopsy, then fixed in formalin and embedded in paraffin. Immunostaining was performed to examine the expression of TS protein. Patient background information is provided in Table I. The patients comprised 78 males and 42 females (mean age, 65.7 years). Additionally, there were 81 patients with adenocarcinoma, 17 with squamous cell carcinoma, 12 with non-small cell carcinoma, 7 with large cell carcinoma and 3 with small cell carcinoma. Eleven patients were positive for the EGFR gene mutation; performance status (PS) was 0-1 in 103 patients; the disease stage was IIIB or IV in 100 patients; and there were 29 non-smokers. The primary treatment for 85 patients $(71 \%)$ was chemotherapy alone, and out of these 85 patients, 50 were administered CbPac combined chemotherapy (Table I).

TS immunostaining. Using the paraffin block of lung cancer tissue collected prior to treatment, the expression of TS protein was examined immunohistochemically. The paraffin block was cut into $10-\mu \mathrm{m}$ sections and stained using immunostaining methods. The sections were then deparaffinized by being treated with $100 \%$ xylene three times for 2 min each, immersed in 99, 90 and $70 \%$ ethanol for 1 min each, washed with running water for 3 min and then reacted in $0.3 \%$ hydrogen peroxide-added methanol at room temperature for $15 \mathrm{~min}$ to inhibit endogenous peroxidase activity. After washing with water for $5 \mathrm{~min}$, the sections were transferred to $0.01 \mathrm{M}$ citrate buffer and treated in a microwave oven for $15 \mathrm{~min}$ to inactivate the antigen. After returning to room temperature, the sections were washed with $0.01 \mathrm{M}$ phosphate-buffered saline (PBS), and allowed to stand in $2 \%$ bovine serum albumin (BSA)/PBS at room temperature for $15 \mathrm{~min}$ to inhibit non-specific reactions. The sections were then incubated with monoclonal mouse anti-TS antibody and diluted 100-fold with PBS as the primary antibody at $4^{\circ} \mathrm{C}$ overnight. Subsequently, the sections were washed with PBS and incubated with peroxidase-labeled dextran 70-conjugated mouse immunoglobulin/goat anti-polyclonal antibody and peroxidase-labeled 

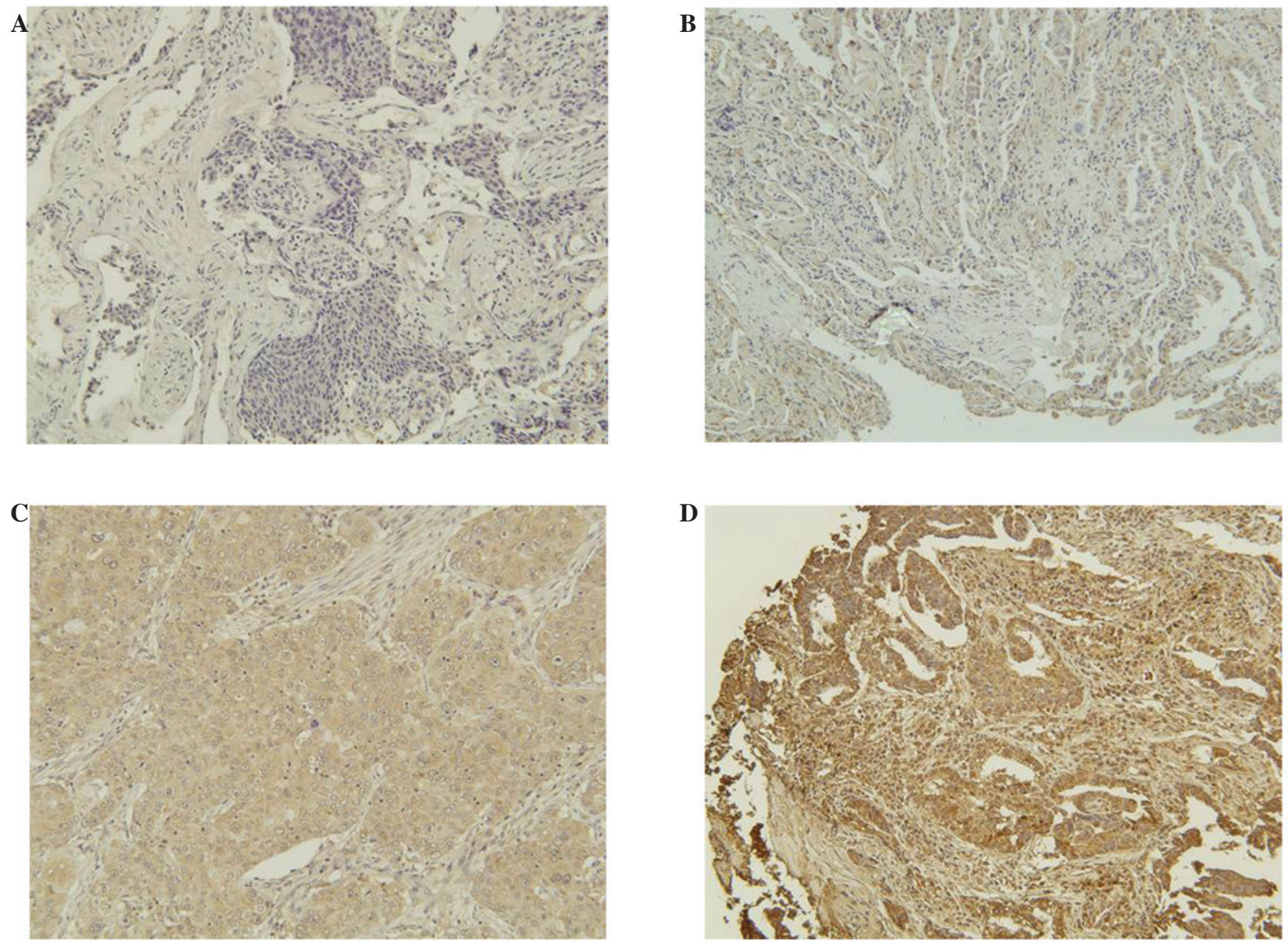

Figure 1. Immunohistochemical staining of human NSCLC tissue. Representative sections of carcinomas showing (A) negative staining, as well as (B) weak, (C) strong and (D) very strong expression of TS. Magnification, x200. NSCLC, non-small cell lung cancer.

dextran 500-conjugated anti-mouse immunoglobulin/goat anti-polyclonal antibody as the secondary antibodies at room temperature for $30 \mathrm{~min}$.

After washing with PBS again, the sections were stained with $0.04 \%$ diaminobenzidine (DAB), nuclear stained with hematoxylin, washed with water, dehydrated with 70, 80, 90 and $99 \%$ ethanol, penetrated with $100 \%$ xylene, encapsulated and examined microscopically.

Evaluation of immunostaining. Expression of TS protein was evaluated using H-scoring. Staining intensity was evaluated as 0 (negative, Fig. 1A), 1 (weakly positive, Fig. 1B), 2 (moderately positive, Fig. 1C) or 3 (strongly positive, Fig. 1D), and multiplied by the proportion (\%) of positive cells to calculate the $\mathrm{H}$-score as previously described (15).

Statistical analysis. To examine the effect of patient background factors, the Mann-Whitney U test was used to compare TS protein expression in the two groups. To evaluate the correlation between TS protein expression and therapeutic effects, the latter were evaluated by investigating the response rate (RR), progression-free survival (PFS) and overall survival (OS). To compare RR between the two groups, the Chi-square test was performed, while the log-rank test was performed using the
Kaplan-Meier method to compare PFS and OS between the two groups. $\mathrm{P}<0.05$ was considered to indicate a statistically significant difference.

\section{Results}

TS protein expression and patient background factors. The mean $\mathrm{H}$-score was $173.1 \pm 99.5$ for adenocarcinoma, $200.0 \pm 71.5$ for squamous cell carcinoma, $193.7 \pm 110.0$ for large cell carcinoma, $188.7 \pm$ ? for non-small cell carcinoma and $266.7 \pm 57.7$ for small cell carcinoma patients. TS expression tended to be higher in small cell carcinoma compared with adenocarcinoma ( $\mathrm{P}=0.0974)$, while no additional significant differences were observed among the other types of lung cancer tissue (Fig. 2A). Regarding the additional background factors, no significant differences in gender, presence/absence of EGFR gene mutation or PS were observed. However, the mean H-score was $150.7 \pm 87.2$ for non-smokers and 192.3 \pm 97.6 for smokers, indicating a significantly higher expression of TS protein in smokers ( $\mathrm{P}=0.0429)$ (Fig. 2B).

TS protein expression and patient survival. The median H-score in NSCLC patients was 200. Patients with a higher $\mathrm{H}$-score were evaluated as the high TS protein expression 
$\mathbf{A}$

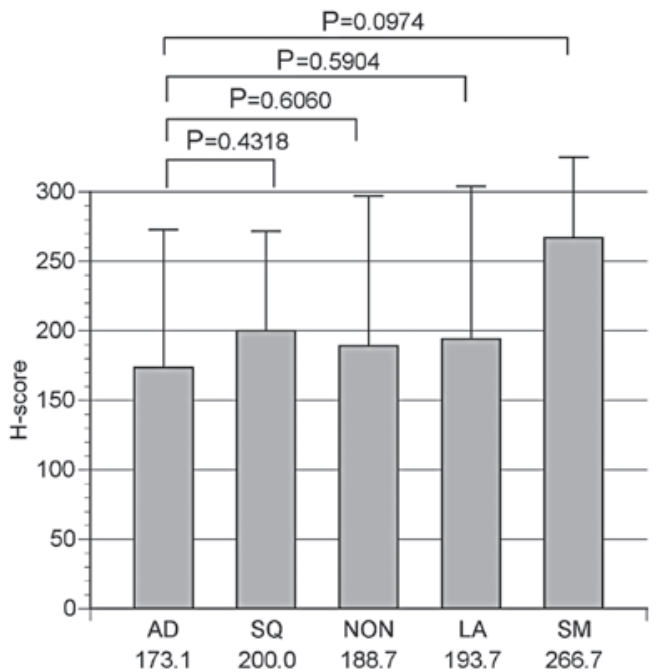

B

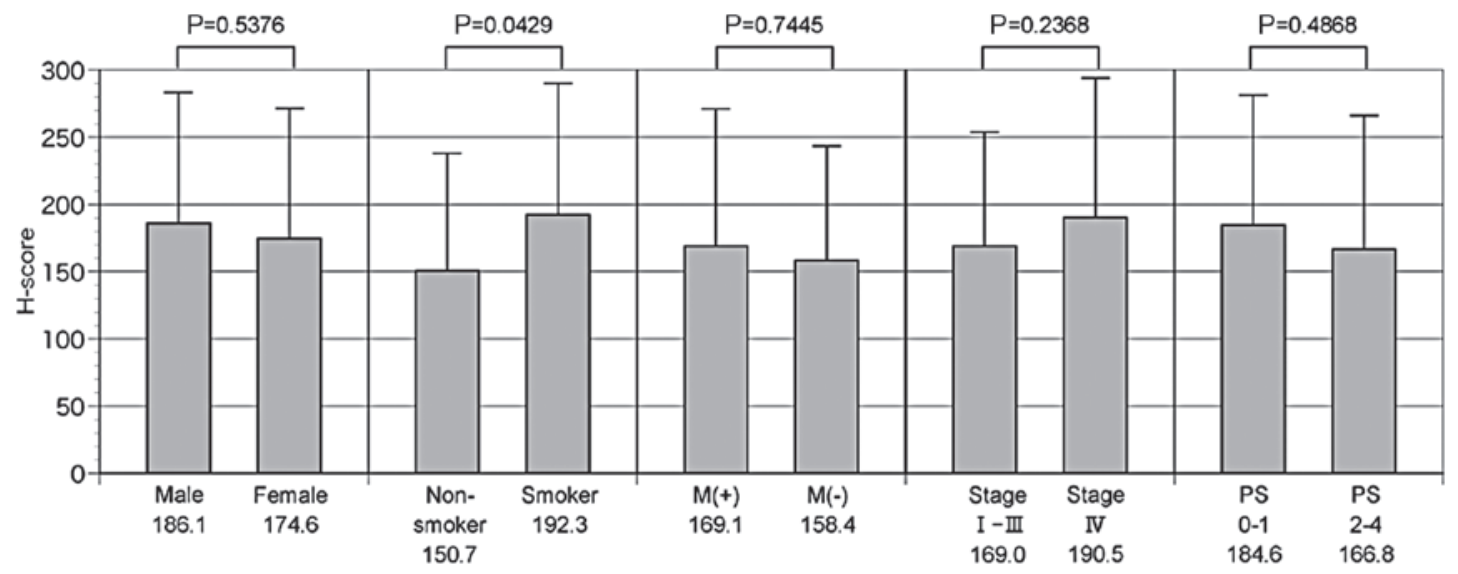

Figure 2. TS protein expression in lung cancer according to (A) tumor and (B) patient characteristics. AD, adenocarcinoma; SQ, squamous cell carcinoma; NON, non-small cell carcinoma; LA, large cell carcinoma; SM, small cell carcinoma; M, EGFR mutation; PS, performance status.

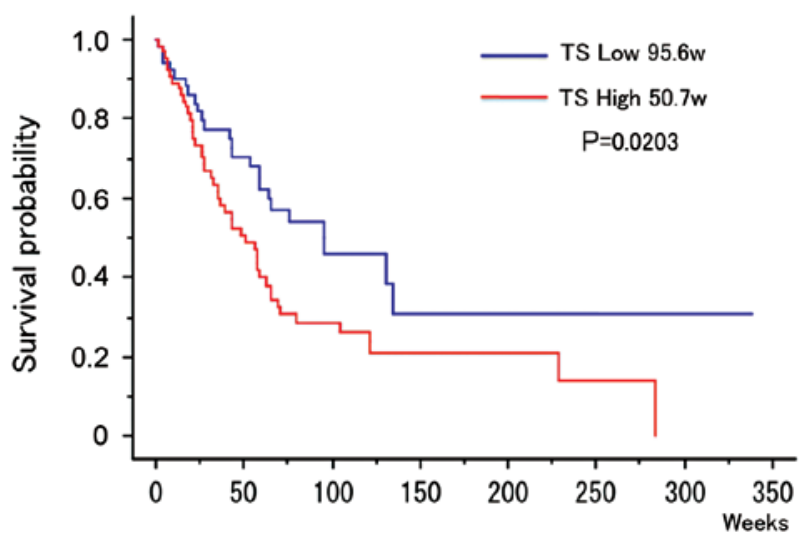

Figure 3. Thymidylate synthase (TS) protein expression of non-small cell lung cancer (NSCLC) according to overall survival.

group $(n=63,53.8 \%)$ and patients with a lower H-score were evaluated as the low TS protein expression group $(n=54$, $46.1 \%$ ). OS (median value) was 95.6 weeks in the low-expression and 50.7 weeks in the high-expression group, indicating a significant prolongation of survival in the low-expression group ( $\mathrm{P}=0.0203)$ (Fig. 3).

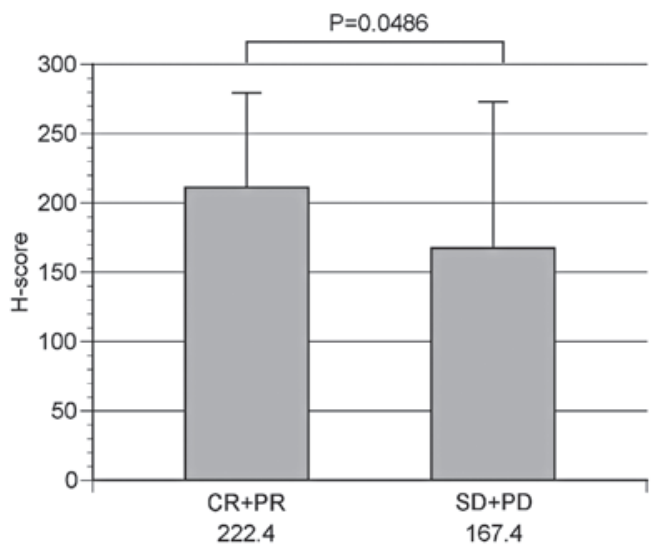

Figure 4. Response rate according to the expression level of TS in non-small cell lung cancer (NSCLC) in patients treated with CbPac. CR, complete response; $\mathrm{PR}$, partial response; $\mathrm{SD}$, stable disease; $\mathrm{PD}$, progressive disease.

TS protein expression and therapeutic effects. NSCLC was observed in 117 patients, 50 of whom were administered $\mathrm{CbPac}$ therapy as the primary treatment. The correlation between TS protein expression and the therapeutic effects of CbPac therapy was, therefore, examined in these 50 patients, 31 of whom $(62.0 \%)$ comprised the high TS protein expression group and 
A

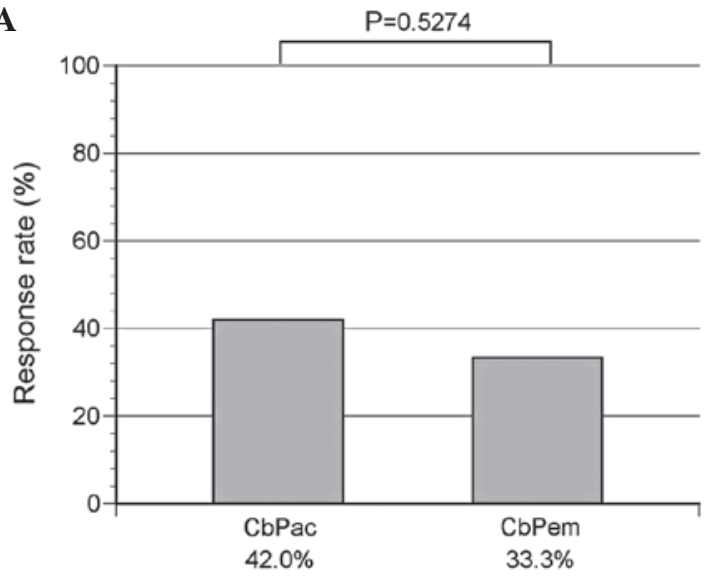

C
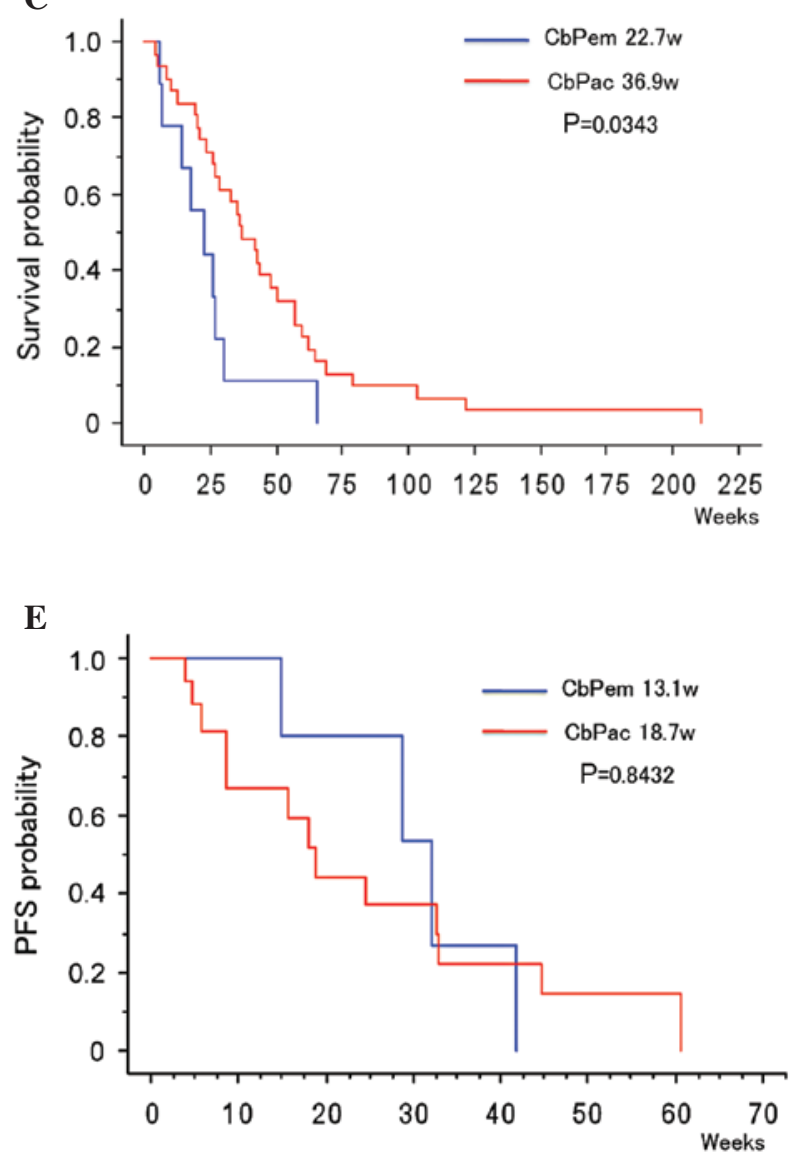

B

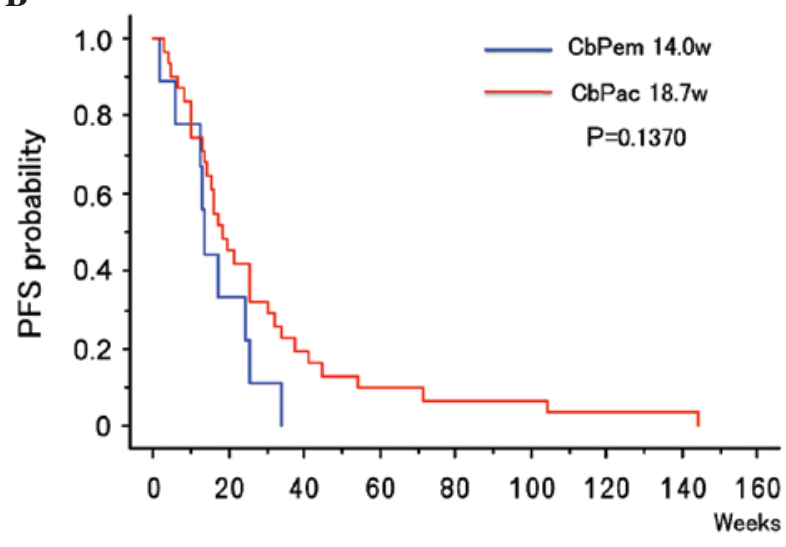

D

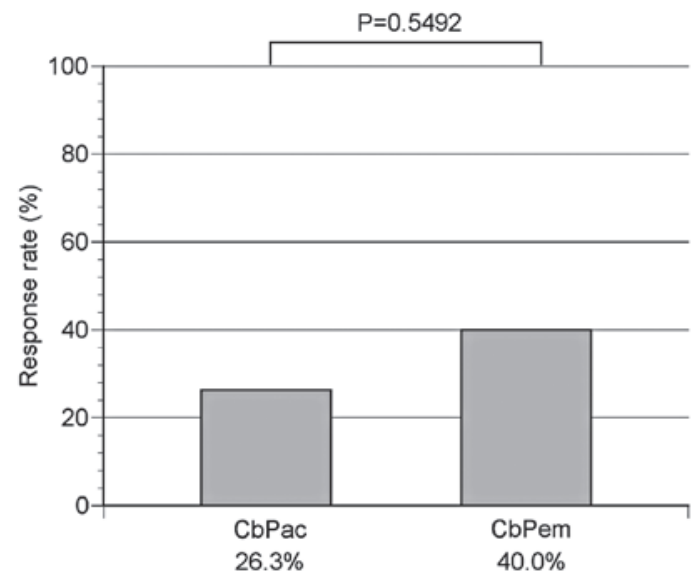

$\mathbf{F}$

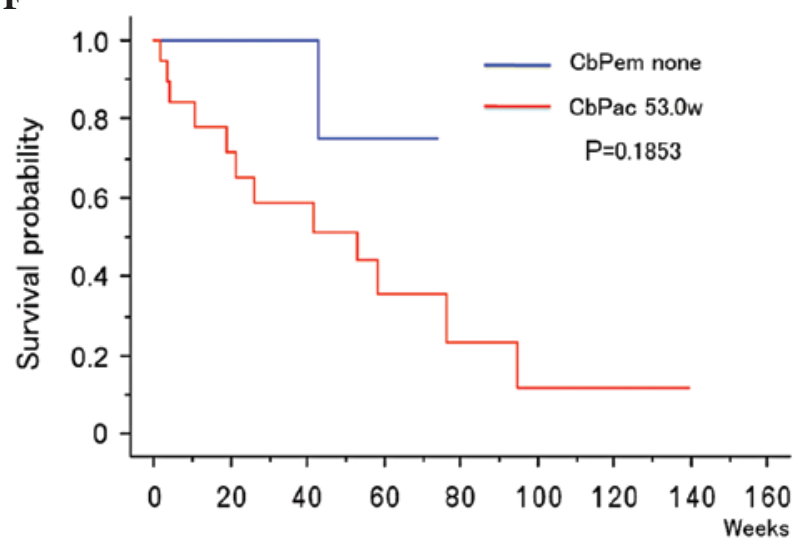

Figure 5. (A) Response rate, (B) progression-free survival and (C) overall survival in non-small cell lung cancer (NSCLC), indicating a high level of TS expression in patients treated with CbPac or CbPem. (D) Response rate, (E) progression-free survival (PFS) and (F) overall survival in NSCLC, indicating a low level of TS expression in patients treated with CbPac or CbPem. CbPac, carboplatin + paclitaxel combined chemotherapy; CbPem, carboplatin + pemetrexed combined chemotherapy.

$19(38.0 \%)$ the low TS protein expression group. Patients whose $\mathrm{RR}$ was complete response $(\mathrm{CR})(\mathrm{n}=0)$ or partial response $(\mathrm{PR})$ $(n=19)$ comprised the response group $(n=19)$, while those whose RR was stable disease (SD) $(n=20)$ or progressive disease (PD) $(n=11)$ comprised the non-response group $(n=31)$. The mean H-score was $222.4 \pm 68.1$ in the response and 167.4 \pm 105.6 in the non-response groups, indicating a significantly higher TS expression in the response group ( $\mathrm{P}=0.0486)$ (Fig. 4).

The therapeutic effects of CbPac (31 patients) and CbPem therapies (9 patients) as the primary treatment were compared in the high TS expression group of NSCLC patients. RR was $42.0 \%$ and $33.3 \%$ in the CbPac and CbPem therapy groups, respectively $(\mathrm{P}=0.5274$, Fig. $5 \mathrm{~A})$. PFS (median value) was 18.7 and 14.0 weeks in the $\mathrm{CbPac}$ and $\mathrm{CbPem}$ therapy groups, respectively ( $\mathrm{P}=0.1370$, Fig. $5 \mathrm{~B})$. OS (median value) was 36.9 and 22.7 weeks in the $\mathrm{CbPac}$ and $\mathrm{CbPem}$ therapy groups, respectively, indicating a significant prolongation of OS in the CbPac therapy group ( $\mathrm{P}=0.0343$, Fig. 5C).

When CbPac (19 patients) and CbPem therapies (5 patients) were compared in the low TS expression group, RR was 26.3 
and $40.0 \%$ in the CbPac and CbPem therapy groups, respectively ( $\mathrm{P}=0.5492$, Fig. 5D). PFS (median value) was 18.7 and 13.1 weeks in the $\mathrm{CbPac}$ and $\mathrm{CbPem}$ therapy groups, respectivley ( $\mathrm{P}=0.8432$, Fig. 5E). OS (median value) was 53.0 weeks in the CbPac therapy group, whereas the median survival period was not achieved in the CbPem group $(\mathrm{P}=0.1853$, Fig. 5F).

\section{Discussion}

The standard treatment for advanced NSCLC is two-drug combination therapy containing a platinum agent, which has been shown to prolong the median survival time from 6 to 8 weeks and improve the 1-year survival rate from 15 to $25 \%$, as previously demonstrated (16). One of the recommended drugs for combination with platinum is Pac, with CbPac therapy constituting one of the standard therapeutic methods for the treatment of lung cancer. Chemotherapy is employed in the treatment of advanced NSCLC. Thus, to increase its efficacy through the selection of the most suitable drugs, predictive factors are being investigated. One predictive factor of the efficacy of platinum agents is the excision repair cross-complementing 1 (ERCC1) gene, a DNA repair protein (17). Class III $\beta$-tubulin is another known prognostic factor of Pac (18). However, whether ERCC1 and $\beta$-tubulin are also predictive factors of the effects of $\mathrm{CbPac}$ therapy on advanced NSCLC remains to be determined.

TS metabolizes 5,10-methylenetetrahydrofolate $\left(\mathrm{CH}_{2} \mathrm{THF}\right)$ as well as deoxyuridylate-5'-monophosphate (dUMP) by reductive methylation to deoxythymidine-5'-monophosphate (dTMP) to produce the thymine nucleotides required for DNA synthesis, and is involved in the biosynthesis of pyrimidine. 5-fluorodeoxyuridine monophosphate (FdUMP), a target enzyme of 5-fluorouracil (5-FU) and an active metabolite of 5-FU, binds to TS and activates folic acid to promote the formation of TS-FdUMP- $\mathrm{CH}_{2} \mathrm{FH}_{4}$ ternary complexes. When TS is completely inhibited, DNA synthesis in cells is also inhibited, resulting in antitumor effects. Accordingly, it has been reported that when the amount of TS protein in tumor cells is high, the antitumor effect of 5-FU is low, and when the amount is small, the sensitivity of 5-FU is high (19). In gastric (19), colon (11), breast (12) and pancreatic cancer (13), the correlation between TS expression and the therapeutic effects of 5-FU and its prognosis has been reported. Additionally, TS protein is positioned downstream of the cell growth signal and is involved in the proliferation of cancer cells. Therefore, the expression of TS protein in cancer cells may influence the effects of various anticancer drugs.

In the present study, we examined the correlation between the expression of TS protein, tissue type, patient background factors, prognosis and the therapeutic effects of CbPac therapy in lung cancer. No significant difference between tissue type and expression of TS protein was identified. However, the TS protein expression was higher in small and squamous cell carcinoma compared with adenocarcinoma, consistent with a previous study (20). Regarding the correlation between patient background factors and TS protein expression, there was no significant difference in gender, smoking status, EGFR gene mutation, clinical stage or PS, while TS protein expression was higher in smokers compared with non-smokers. Since smoking has been shown to induce mutations in genes such as p53 and KRAS (21-23), it is likely that this increase in TS protein expression was the result of a gene mutation induced by smoking. In addition, significant prolongation of OS was observed in the low compared with the high TS expression group, suggesting that TS protein expression may affect the prognosis of lung cancer. The rate of expression of TS protein in the primary lesion has been reported to correlate with malignancy (24), and is also thought to be involved in the biological malignancy of cancer.

The investigation of the correlation between the therapeutic effects of CbPac therapy and expression of TS protein demonstrated a higher TS in the response compared with the non-response group. This suggests that a higher TS expression is closely associated with higher efficacy of CbPac therapy. Moreover, when the effects of $\mathrm{CbPac}$ and $\mathrm{CbPem}$ therapies were compared in the high TS expression group, RR tended to be higher and PFS longer in the CbPac therapy group. OS was also significantly prolonged in the CbPac therapy group. These results suggest that $\mathrm{Pac}$ is more effective compared with Pem in the treatment of NSCLC with high TS expression, suggesting a higher efficacy of CbPac therapy in the high TS expression group, which is in contrast to the correlation between the amount of TS protein and the antitumor effect of 5-FU. As mentioned previously, 5-FU inhibits DNA synthesis to achieve antitumor effects. Similarly, Pem also inhibits DNA synthesis by inhibiting TS, a folate metabolic enzyme, to achieve antitumor effects. However, Pac stops cell division resulting in antitumor effects by inhibiting and stabilizing depolymerization of microtubules in the $\mathrm{M}$ phase of cell division. Since TS is a rate-limiting enzyme of DNA synthesis, a high TS expression is thought to indicate a high rate of cell division.

When CbPac and CbPem therapies were compared in the low TS expression group, RR was higher, while PFS and OS tended to be prolonged in the CbPem therapy group. In the low TS expression group, Pem was more effective compared with Pac. It has been reported (20) that Pem inhibits the growth of tumor cells and induces cell death mainly through the inhibition of TS, resulting in antitumor effects. Pem also shows high sensitivity in low TS-expressing cells. Therefore, Pem likely has an impact on NSCLC with low TS expression.

In the present study, expression of TS protein was examined using immunostaining methods. While quantification of mRNA can also be used to examine protein expression, immunostaining requires few specimens and is a simple procedure, constituting a convenient test method in clinical practice. Currently, the standard therapeutic method for primary treatment of NSCLC is CbPac + bevacizumab or cisplatin $(\mathrm{Cis})+$ Pem. Therefore, prediction of the therapeutic effects of Pac and Pem is of high clinical relevance. Concerning the results of the present study, determination of TS protein expression using immunostaining methods is considered useful for the selection of Pac- or Pem-based platinum doublet during treatment. The findings also suggest that the selection of an effective primary treatment according to the rate of TS protein expression in lung cancer tissues may lead to improved prognosis, as observed in EGFR gene mutation analysis. 


\section{References}

1. Lynch TJ, Bell DW, Sordella R, et al: Activating mutations in the epidermal growth factor receptor underlying responsiveness of non-small-cell lung cancer to gefitinib. N Engl J Med 350: 2129-2139, 2004

2. Paez JG, Janne PA, Lee JC, et al: EGFR mutations in lung cancer: correlation with clinical response to gefitinib therapy. Science 304: 1497-1500, 2004.

3. Rosell R, Monzo M, Molina F, et al: K-ras genotypes and prognosis in non-small-cell lung cancer. Ann Oncol 6: S15-S20, 1995.

4. Takano T, Fukui T, Ohe Y, et al: EGFR mutations predict survival benefit from gefitinib in patients with advanced lung adenocarcinoma: a historical comparison of patients treated before and after gefitinib approval in Japan. J Clin Oncol 26: 5589-5595, 2008.

5. Mok TS, Wu YL, Thongprasert S, et al: Gefitinib or carboplatin-paclitaxel in pulmonary adenocarcinoma. N Engl J Med 361: 947-957, 2009.

6. Ferguson PJ, Collins O, Dean NM, et al: Antisense down-regulation of thymidylate synthase to suppress growth and enhance cytotoxicity of 5-FUdR, 5-FU and Tomudex in HeLa cells. Br J Pharmacol 127: 1777-1786, 1999.

7. Flynn J, Berg RW, Wong T, et al: Therapeutic potential of antisense oligodeoxynucleotides to down-regulate thymidylate synthase in mesothelioma. Mol Cancer Ther 5: 1423-1433, 2006.

8. Lin SB, Ts'o PO, Sun SK, et al: Inhibition of thymidylate synthase activity by antisense oligodeoxynucleotide and possible role in thymineless treatment. Mol Pharmacol 60: 474-479, 2001

9. Hann N, Shepherd FA, Fossella FV, et al: Randomized phase III trial of pemetrexed versus docetaxel in patients with non-small-cell lung cancer previously treated with chemotherapy. J Clin Oncol 22: 1589-1597, 2004.

10. Okamoto I, Yoshioka H, Morita S, et al: Phase III trial comparing oral S-1 plus carboplatin with paclitaxel plus carboplatin in chemotherapy-naive patients with advanced non-small-cell lung cancer: result of a West Japan oncology group study. J Clin Oncol 20: 5240-5246, 2010.

11. Shirota Y, Stoehlmacher J, Brabender J, et al: ERCC1 and thymidylate synthase mRNA level predict survival for colorectal cancer patients receiving combination oxaliplatin and fluorouracil chemotherapy. J Clin Oncol 19: 4298-4304, 2001.

12. Nishimura R, Nagao K, Miyayama H, et al: Thymidylate synthase levels as a therapeutic and prognostic predictor in breast cancer. Anticancer Res 19: 5621-5626, 1999.
13. Takamura M, Nio Y, Yamasawa K, et al: Implication of thymidylate synthase in the outcome of patients with invasive ductal carcinoma of the pancreas and efficacy of adjuvant chemotherapy using 5-fluorouracil or its derivatives. Anticancer Drugs 13: 75-85, 2002.

14. Zheng Z, Li X, Schell MJ, et al: Thymidylate synthase in situ protein expression and survival in stage I non small-cell lung cancer. Cancer 112: 2765-2773, 2008

15. Chen CY, Chang YL, Shin JY, et al: Thymidylate synthase and dihydrofolate reductase expression in non-small cell lung carcinoma: the association with treatment efficacy of pemetrexed. Lung Cancer 74: 132-138, 2011.

16. Non-small Cell Lung Cancer Collaborative Group: Chemotherapy in non-small cell lung cancer: a meta-analysis using updated date on individual patients from 52 randomized clinical trials. BMJ 311: 899-909, 1995.

17. Olaussen KA, Dunant A, Fouret P, et al; IALT Bio Investigators: DNA repair by ERCC1 in non-small cell lung cancer and cisplatin-based adjuvant chemotherapy. N Eng J Med 355: 983-991, 2006.

18. Yin S, Bhattacharya $\mathrm{R}$ and Cabral F: Human mutations that confer paclitaxel resistance. Mol Cancer Ther 9: 327-335, 2010.

19. Lenz HJ, Leichman CG, Danenberg KD, et al: Thymidylate synthase mRNA level in adenocarcinoma of the stomach: a predictor for primary tumor response and overall survival. J Clin Oncol 14: 176-182, 1996.

20. Ceppi P, Volante M,Saviozzi S, et al: Squamous cell carcinoma of the lung compared with other histotypes shows higher messenger RNA and protein levels for thymidylate synthase. Cancer 107: 1589-1596, 2006.

21. Suzuki H, Takahashi T, Kuroishi T, et al: p53 mutations in non-small cell lung cancer in Japan: association between mutations and smoking. Cancer Res 52: 734-736, 1992.

22. Ahrendt SA, Decker PA, Alawi EA, et al: Cigarette smoking is strongly associated with mutation of the K-ras gene in patients with primary adenocarcinoma of the lung. Cancer 92: 1525-1530, 2001.

23. Mitsudomi T, Oyama T, Nishida K, et al: Loss of heterozygosity at $3 \mathrm{p}$ in non-small cell lung cancer and its prognostic implication. Clin Cancer Res 2: 1185-1189, 1996.

24. Johnston PG, Fisher ER, Rocktte HE, et al: The role of thymidylate synthase expression in prognosis and outcome of adjuvant chemotherapy in patients with rectal cancer. J Clin Oncol 12: 2640-2647, 1994. 